Review

\title{
Current Understanding on Tail Regeneration in Green Anoles (Anolis carolinensis)
}

\author{
Zulkar Nain *, Md Ariful Islam, Sadrul Hasan Chowdhury and Sadia Afroza \\ Department of Biotechnology and Genetic Engineering, Faculty of Applied Science and Technology, \\ Islamic University, Kushtia 7003, Bangladesh; arif.gbt.bd@gmail.com (M.A.I.); \\ sadrul.chowdhury23@gmail.com (S.H.C.); sadia.iuk@gmail.com (S.A.) \\ * Correspondence: zlkr.bd@gmail.com; Tel.: +88-01911-073860
}

\begin{abstract}
Regeneration of lost tail is of great importance to lizards. Anolis carolinensis, a green lizard, is capable of regenerating its tail efficiently after autotomy. Hence, it is considered as a model organism in regeneration study. A. carolinensis shed its tail in order to distract the predator's attention and thus makes a way to escape. Restoring of the amputated tail takes several days and the mechanism is currently clearly understood. Although save its life, tail regeneration is associated with the impairment of several vital functions in Anoles. In addition, various differences have been observed between original and regenerated tail in terms of mechanism and structure. To date, very little work has been conducted on tail autotomy and regeneration at molecular and genetic level. The genes responsible for regeneration in anoles are identified recently. These genes are evolutionarily conserved through all tetrapod vertebrates. They are, however, in a state of 'switched-off' in other vertebrates including humans. Consequently, a throughout study of these so called 'switched-off' genes may provide a way of restoring lost organs in human, and thus could revolutionize the modern medical science.
\end{abstract}

Keywords: green anole; tail autotomy; regeneration, microRNAs, Anolis carolinensis

\section{Introduction}

Autotomy or self-amputation is a voluntary shedding of caudal limb or tail as an anti-predation strategy. This phenomenon can be found in both vertebrate and invertebrate but particularly common in lizards [1,2]. The evolution of lizards provides them this remarkable ability to selfamputate their tails when threatened $[3,4,5]$ and regenerate it again later [6, 7]. Among vertebrate, tail is the only appendages that can be released as anti-predation strategy. The detached tail keeps wiggling about 30 minutes [1, 8], fueled by anaerobic metabolism [8], distract the attention of the predator and thus provides an escaping maneuver for lizards $[1,8]$.

Anolis carolinensis is a prominent non-avian reptilian lizard (see Figure 1), commonly known as green anole, which can regenerate their tails efficiently after self-amputation. This makes green anole an excellent reptilian model for studying regeneration [9], muscular morphology and genomics [10]. The complete genome of Anolis carolinensis was published recently in 2011 [11, 12]. Whole genome mapping has greatly facilitated studies of tail regeneration and developmental stages in green anoles as well as other vertebrates at gene level [10-12]. Therefore, the tail regeneration of Anoles is being recognized among biomedical scientists for the study of wound healing and multi-tissue recuperation [2] in highly ordered organisms. 


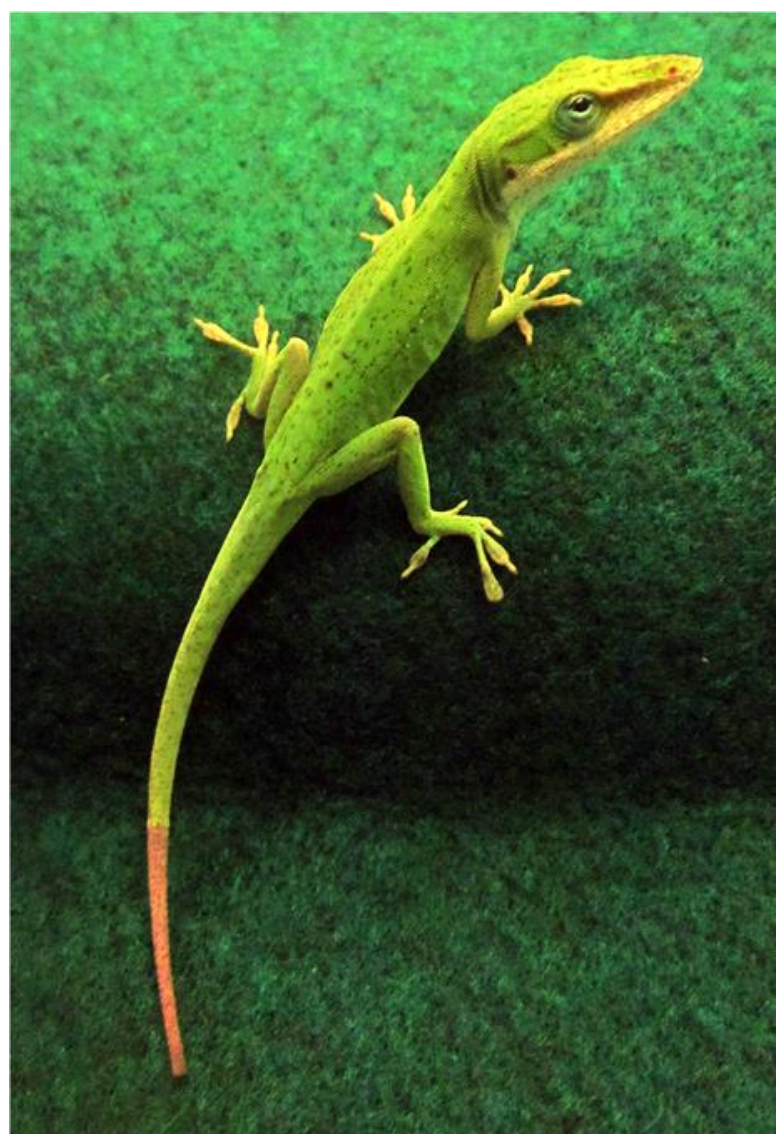

Figure 1: Male Anolis carolinensis specimen with a regenerated tail marked as brown color [13].

Previously, genetic studies of developmental process were mainly based on frog, mouse or zebrafish models [14]. Now reptiles, particularly lizards, are coming into the mainstream because of their diversity and unique feature of regeneration. Among lizards, green anoles are the most important in tail regeneration studies [3, 6, 15-19]. Evolved from non-avian reptilian lineage, which is also the ancestral origin of mammals, green anoles share their genome with mammals [12]. This common amniotic ancestral origin implies that regenerative capability of functional appendages is deeply homologous in animals but it is not conserved uniformly in vertebrates. Despite of being the closest relatives to human [20], lizards can regenerate their tails (i.e., green anoles) and limbs (i.e., salamander [21]) even in their adulthood while human cannot. This indicates that ancestral vertebrates have this ability but mammals (i.e., humans) lost it somehow during their evolution. Perhaps the genes responsible for regeneration had "shut off" during the evolution of mammals while remaining intact in lizards [14]. Molecular mechanism of regeneration process in lizards may shed light on the latent regenerative capabilities in mammals. Mapping and sequencing of mRNA present in regenerating tails of Anolis carolinensis, indicates conserved gene activation during regeneration [20]. If the genes and pathways responsible for regeneration were conserved in mammals like lizards, in future it could be possible to activate the genes, thereby harness the mechanisms in mammals, even in humans [21].

\section{Evolutionary Aspects of Autotomy and Regeneration}

Caudal autotomy of lizards can be traced to Lepidosuaria, a common ancestor of all lizards. Tail autotomy plays an essential role in survival of various lizards [22]. They initiate autotomy when seized or poisoned by a predator during predator-prey interactions [3]. In lizards, natural tail shedding followed by regeneration of the lost tail is pretty much common. Shed tail wiggle for sometimes after amputation. This helps to outface the predators and thus protects its head or other vital body parts [23]. 
In evolution, survival is all that ever matters which defines Darwin's "struggle for the existence" but to exist such a sacrificing strategy (e.g., autotomy) in nature, there must be a potential benefit over the loss. The potential costs of autotomy must be weighed against the benefits of this strategy [24]. Therefore, the costs of self-amputation should be less than benefits or least to be merged. Appendages may be lost when they have been envenomated as a method of removing the toxin or venom from the circulatory system [5]. To minimize or compensate the loss of tail shedding, lizards have evolved specialized behavioral and physiological adaptation practices i.e., regeneration of lost tails [24]. Furthermore, multiple advantages associated with self-amputation. For instance, shed tails move continuously or release toxin to distract or kill predators. Besides, it can be used as a substitute meal. Postautotomy survival, however, greatly depends on the species, anti-predation strategy, size of shed limb and escape speed after autotomy [5, 23]. Moreover, the study of autotomy should be done in touch of both lizards experienced autotomy without regeneration and lizards experienced autotomy with regeneration to understand the evolutionary significance [5].

The environment, where lizards live, plays a significant role in tail autotomy. According to Johannes Foufopoulos, ability and rapidity of tail shedding vary with different species and environments. In his opinion, autotomy in lizards developed according to the presence of predators (i.e., vipers) during evolution. For instance, lizards in mainland areas or on islands with no vipers mostly incapable of shed their tails. On the contrary, lizards living with many and few predators have instant ability and difficulty of tail shedding, respectively. Birds or mammals have to catch the lizards before eating but vipers only need to inject a tiny amount of their venom with their fangs. By instant shedding of tail, damage of vital organs due to poisonous venom can be avoided. Therefore, tail autotomy in lizards probably developed to dodge the venomous attack of vipers. In Foufopoulos words, "you may lose your tail but you could save your life" [25]. It helps us understand natural tail shedding and its regeneration were coevolved during the course of their evolution.

\section{Mechanism of Tail Regeneration in Anolis carolinensis}

The regeneration of tail in Anoles has extensively been studied in past several decades. Therefore, the basic pathway of tail regeneration in Anolis carolinensis is now apparently clear [2]. Histologic and autoradiographic studies suggest a regeneration process where (1) muscle contributes a very little in tail regeneration; (2) connective tissue elements are the primary source of cells for regeneration to occur; (3) blastema is not found in tail tip unlike amphibians. This indicates tail regeneration in amphibians and lizards is not same in terms of blastema formation [26]. The whole regeneration process can take up to 60 days-post-autotomy (dpa) with a specific end point. The overall process can be divided into four stages, namely, wound healing, mesenchymal cone formation, tail regrowth and maturation, with certain timeframe (see Table 1, Figure 2) [6, 20]. Cell death, dedifferentiation and stem cell proliferation play central role in these stages. Furthermore, specific signal of polarity and positional identity for preexisting body structure must be combined in newly regenerated tissues. Gene knockdown, functional analysis and transgenesis based lineage techniques were the key tools to understand the process clearly [27].

Table 1. Time requires in four main stages of post autotomy during tail regeneration ${ }^{1}$

\begin{tabular}{cc}
\hline Principal Stages & Required Time \\
\hline Wound Healing & Up to $10 \mathrm{dpa}^{2}$ \\
Cone Formation & From 10 to $15 \mathrm{dpa}$ \\
Tail Regrowth & From 15 to $25 \mathrm{dpa}$ \\
Maturation of Tail & From 25 to $60 \mathrm{dpa}$ \\
\hline${ }^{1}$ This timeline is prepared from the data described in Hutchins et al., 2014 [20] \\
${ }^{2}$ dpa is the abbreviated form of days-post-autotomy.
\end{tabular}




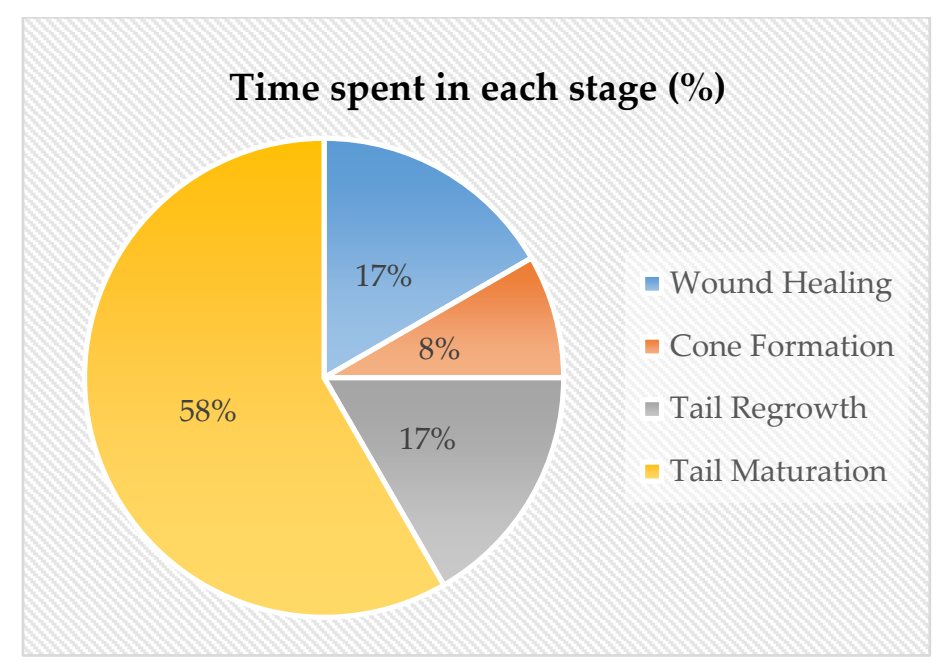

Figure 2. Time required in each step: cone formation needed the least amount of time $(8 \%)$, wound healing and tail regrowth both needed same amount of time (17\%), whereas, maturation needed more than half of the total time (58\%). This pie-chart is prepared based on Table 1 [20].

The earlier stages of tail regeneration are characterized by tissue patterning and differentiation. First 10 days are featured by wound healing (0-10 days-post-autotomy (dpa), see Figure 3B). Within $10 \mathrm{dpa}$, blastema like structure with enclosed blood vessels are formed at wound site. Outgrowth is not noticeable still by this time. In fact, blastema like wound epithelium initiates the first visible outgrowth through the formation of mesenchymal cone during 10-15 dpa. After $15 \mathrm{dpa}$, highly vascularized tissue and myofibrils are visualized (see Figure 3C). During 15-20 dpa, mesenchymal cone developed into central cartilage tube and myofibrils into surrounding skeletal muscle (see Figure 3D). Tail tip still remains vascular at this stage (see Figure 3B-D). By $25 \mathrm{dpa}$, visual lengthening of regenerating tail and formation of muscle and cartilage covering the ependymal core can be observed (see Figure 3E). Continuous tail outgrowth and differentiation are spotted after $25 \mathrm{dpa}$; and maturation stage continue until $60 \mathrm{dpa}$. No significant outgrowth is marked after $60 \mathrm{dpa}$. However, myosin heavy chain (MHC) is found along the length of the developing tail, except distal tail tip (see Figure 3F) [20].

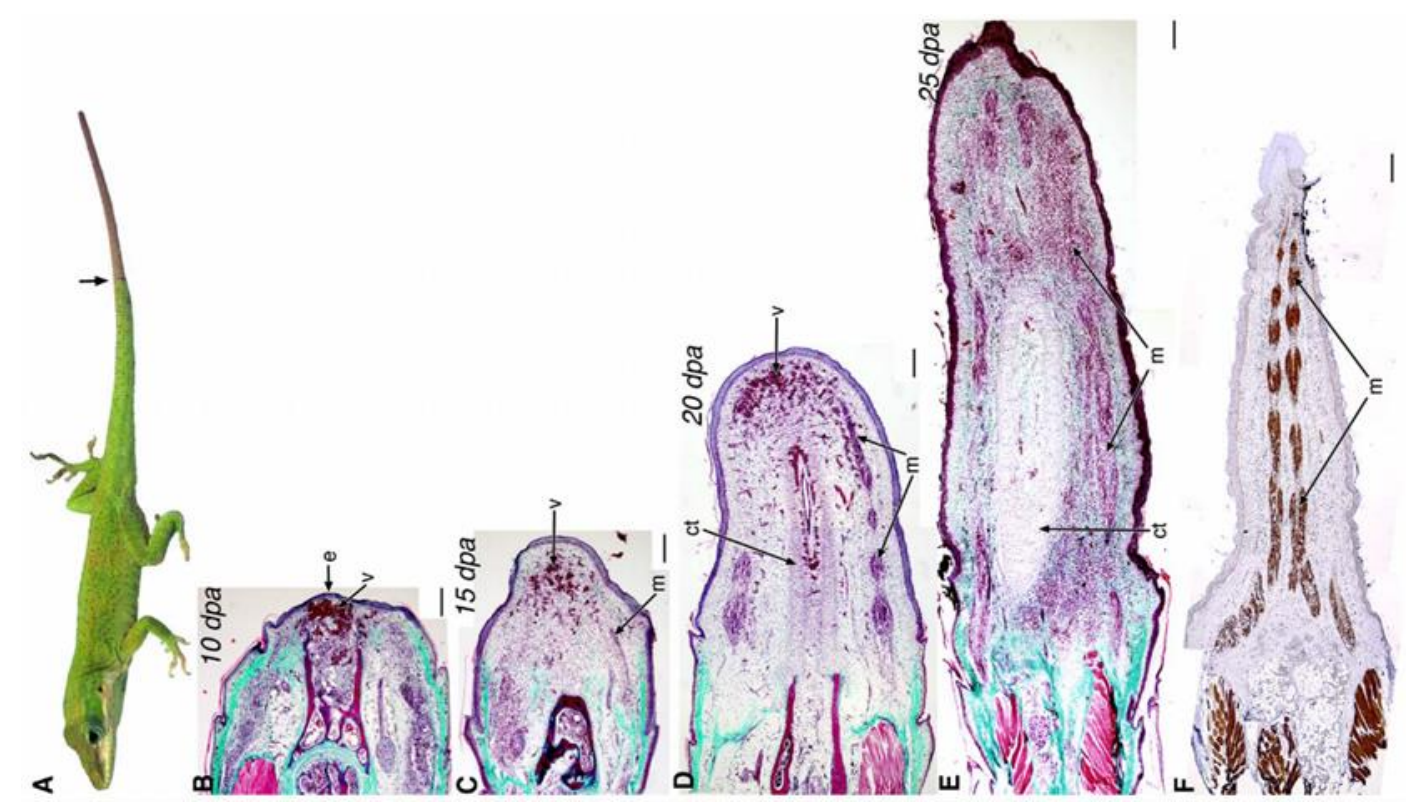

Figure 3. Histological depiction of successive stages of tail regeneration in Anoles at (B) 10, (C) 15, (D) 20 and (E) 25 dpa. Muscle, keratin and cytoplasm are stained red, connective tissue and 
collagen are stained green-blue and nuclei are stained black using Gomori's trichrome staining. Immunochemistry of myosin heavy chain represented in the 25 dpa regenerating tail. Here, e, v, m and ct stand for wound epithelium, blood vessels, muscle and cartilaginous tissue, respectively [20].

Immediately after the loss of tail, some structural arrangements occur through the reepithelialization to reduce blood loss and trauma of adjacent tissues. Normally cutting-end ends with infections but in case of Anolis lizards, no infection occurs after amputation. This anti-microbial barrier in the exposed tissues of the tail stump may be due to the presence of $\beta$-defensin (AcBD15) at the wound site unlike normal tissues [28]. Early phase of wound healing comprises of leukocytic response (limited inflammation) and blastema (mass of proliferating mesenchymal-like cells) formation, which later initiates the regeneration of tail. With the extension of blastema, new blood vessel and spinal cord are regenerated within it. Mature hemopoietic cells, which provide immunity and replaced by mesenchymal cells later, are found in the blastema by 5-bromodeoxyuridine (5BrdU) and tritiated thymidine (3H-T) labelling [29]. Together with necessary blood vessel and spinal cord, formation and rearrangement of muscles form a new tail [2]. A hyaline cartilage tube is formed which contains a spinal cord with an ependymal cell core. Myofibers (or myomeres) are produced from myoblasts adjacent to the cartilage tube which is further developed into the layer of muscles beneath dermis of regenerating tail [30,31]. To sum up, an inflammatory response with continuous modulated balance between factors promoting local inflammation and blastema formation is needed prior to the initiation of regeneration process [21].

Among vertebrates, mammals cannot regenerate cartilaginous tissue but lizards can [32]. Administration of 5-BrdU and 3H-T reveals that cartilage tissue of regenerated tail derives from residential stem cells present nearby vertebrae including perichondrium [32]. Newly formed spinal cord comprises of ependymal tube, central nerve fibers and connective tissues. Ependymal tube is a single entity and separate from connective tissues by foramina. Ependymal tissue is composed of epithelial cells [15]. Three different types of cells, namely, ependymal cells (most frequent), glial cells (precursor of neuron) and neuroblasts, are spotted by incorporating $3 \mathrm{H}-\mathrm{T}$ into regenerating tail of Anolis lizards [33]. They found that synapses were formed after 3 weeks of spinal cord regeneration [33]. However, cartilage formation limited the appositional growth in their primary stage. After the formation of an inner and outer perichondrium, the diameter of cartilage tube is maintained through cellular integration to cartilaginous tube and cellular degeneration of the calcifying zones [18].

Scales formation is a part of tail regeneration in Anolis carolinensis. After tail autotomy, de novo scales regenerate from tail blastema, though embryonic and adult scale formation process is different [34]. Scales formation started earlier from 26 post-wound-day (pwd) but scales can be seen throughout the tail, except the tip, from 33 pwd [34] and after two months (about 60 pwd) mature scales of irregular shape and size can be found. Fibroblast Growth Factor7 (FGF7) is found to be associated with beta cell differentiation in epidermal layers during regeneration of scales [35]. Scales of Anolis carolinensis contain three types of beta-proteins (Ac37, Ac39 and Ac40) in their epidermis. Beta proteins Ac37, Ac39 and Ac40 found in mature alpha layer, beta layer of tail, and mature layer of most scales and dewlap, respectively. Beta proteins together with alpha keratins form the denser areas of mature beta layer [36]. However, regenerated scales lose the ability of physiological color change due to the lack of melanophores, precisely iridophores [37].

\section{Conserved MicroRNAs Could Be Involved in Tail Regeneration}

Recent transcriptomic analysis shed light on this aspect. Primary analysis of RNA-Seq from different sections of regenerating tails of Anolis lizards, reveals that 326 differentially expressed genes (see Table 2) are involved in repair mechanism, especially in wound healing, hormonal regulation, muscle development, Wnt and MAPK/FGF pathways. Of total 326 genes, 302 have precise mammalian orthologs and involved predominantly in skeletal muscle development [20]. Differentially expressed genes related to Wnt signaling and lizard tail tip regeneration also show similarity with regeneration of salamander tail tip and mouse digit tip. This suggests that genes responsible for regeneration is conserved in all tetrapod vertebrates [14]. 
Table 2. Selected genes in tail regeneration identified by transcriptomic analysis ${ }^{1}$

\begin{tabular}{|c|c|}
\hline Stages & lved in Specific Processes \\
\hline Myogenesis & $\begin{array}{l}\text { Muscle Contraction (mybpc2, tnnc2, tnnc1, myl3, mybpc1, mybpc3, myl1, pgam2, myot, des, } \\
\text { myom2, myl6b, myom1, chrna1, scn5a, dtna, kcnma1, actc1, acta1, actn2, myh6, tnni2, trdn, } \\
\text { tnnt3, tnnt1, ryr1, stbd1, chrne, casq2, chrng); Muscle Organ Development (mef2c, myod1, } \\
\text { myl2, tnnc1, myl3, mybpc3, myl1, trim72, speg, myl6b, pax7, obsl1, mkx, mkl2, chrna1, actc1, } \\
\text { acta1, mstn, mylpf, myh6, csrp3, flnb, murc, neb, xirp1, itga7, vgll2, tcf15); Skeletal Muscle } \\
\text { Tissue Development (myod1, acta1, myl3, myl6b, pax7, mylpf, vgll2, chrna1, csrp3); Muscle } \\
\text { Cell Differentiation (myod1, actc1, acta1, xirp1, myl2, speg, lgals1, obsl1, myh6, mkl2, chrna1); } \\
\text { Musculoskeletal Movement (tnnt3, tnnt1, tnnc2, tnnc1, chrna1, tnni2); Actin Filament- } \\
\text { Based Process (actc1, tnxb, myl2, acta1, myl1, pdlim3, myh6, gas7, flnb, xirp1, xirp2, myl6b, } \\
\text { limch1, obsl1); Cell Adhesion (hapln1, tnxb, mybpc2, clstn2, egfl6, lpp, mybpc1, col22a1, } \\
\text { mybpc3, col28a1, mgp, actn2, col2a1, actn3, ecm2, col9a1, itga7, acan, susd5, col11a2, thbs4); } \\
\text { Skeletal System Development (bmp3, col9a1, col9a2, tbx15, lect1, clec3a, pax7, acan, mgp, } \\
\text { col2a1, col11a2, tcf15); Extracellular Matrix Organization (csgalnact1, tnxb, adamts20, acan, } \\
\text { col2a1, col11a2, ecm2); Cytoskeleton-Dependent Intracellular Transport (actc1, myl6b, } \\
\text { myl1, myh6); Cellular Ion Homeostasis (kcnma1, jph2, xirp1, pygm, atp2a1, ryr1, chrna1, } \\
\text { chrne, csrp3, sypl2, chrng). }\end{array}$ \\
\hline Chondrogenesis & $\begin{array}{l}\text { Cartilage Development (bmp3, col9a1, lect1, pax7, acan, mgp, col2a1, col11a2); Chondrocyte } \\
\text { Differentiation (col9a1, acan, col2a1, col11a2); Cartilage Condensation (acan, mgp, col2a1). }\end{array}$ \\
\hline $\begin{array}{l}\text { Musculoskeletal } \\
\text { Activity }\end{array}$ & $\begin{array}{l}\text { Regulation of ATPase Activity (tnnt3, myl3, tnnc1, mybpc3, myh6); Proteoglycan } \\
\text { Metabolic Process (csgalnact1, lect1, acan, col2a1). }\end{array}$ \\
\hline $\begin{array}{l}\text { Biological } \\
\text { Adhesion }\end{array}$ & $\begin{array}{l}\text { Biological Adhesion (hapln1, tnxb, mybpc2, clstn2, egfl6, lpp, mybpc1, col22a1, mybpc3, } \\
\text { col28a1, mgp, actn2, col2a1, actn3, ecm2, col9a1, itga7, acan, susd5, col11a2, thbs4). }\end{array}$ \\
\hline & $\begin{array}{l}\text { Response to Wounding (pcsk1, scube1, pdgfra, pla2g7, entpd1, ptx3, mdk, igfbp4, f2r, spp1); } \\
\text { Response to Hormone Stimulus (cga, pcsk1, krt19, tnfrsf11b, bsg, th, pdgfra, spp1); Wnt } \\
\text { Receptor Signaling Pathway, Calcium Modulating Pathway (wnt5a, wnt16, ror2); Wnt }\end{array}$ \\
\hline Wound & Receptor Signaling Pathway ( $d k k 2$, wnt5a, wnt16, ror2, wif1); Cell Surface Receptor \\
\hline Response & $\begin{array}{l}\text { Signaling Pathway (wnt5a, cga, edn3, fgfr4, il1r1, wnt16, gpr158, bsg, maml2, ptpn22, thy1, } \\
d k k 2 \text {, ednra, or5v1, pdgfra, ror2, wif1, pdgfc, entpd1, f2r) Response to Organic Substance } \\
\text { (ednra, cga, pcsk1, krt19, il1r1, tnfrsf11b, bsg, th, pdgfra, f2r, spp1); Inflammatory Response } \\
\text { (scube1, pla2g7, ptx3, igfbp4,f2r, spp1). }\end{array}$ \\
\hline $\begin{array}{l}\text { Hormonal } \\
\text { Regulation }\end{array}$ & $\begin{array}{l}\text { Positive Regulation of Transport (ednra, edn3, pcsk1, rab8b, ptx3, f2r, thy1); Regulation of } \\
\text { Homeostatic Process (ednra, tnfrsf11b, f2r, spp1, thy1); Thyroid Hormone Generation (cga, } \\
\text { dio2). }\end{array}$ \\
\hline $\begin{array}{l}\text { Embryonic } \\
\text { Morphogenesis }\end{array}$ & $\begin{array}{l}\text { Skeletal System Development (wnt5a, tnfrsf11b, pdgfra, ror2, mepe, cbfb, igfbp } 4, \text { spp1, twist } 1 \text { ) } \\
\text { Tube Development (wnt5a, ednra, fgfr4, sall1, pdgfra, ptk7, twist1); Embryonic } \\
\text { Morphogenesis (wnt5a, sall4, th, ptk7, ror2, twist1, ptprq). }\end{array}$ \\
\hline $\begin{array}{l}\text { Immune } \\
\text { Response }\end{array}$ & \\
\hline
\end{tabular}

${ }^{1}$ This table is reproduced from the data generated in transcriptomic analysis done by Hutchins et al. 2014 [20].

Like other vertebrate models, MicroRNAs also contribute to the development of tail regeneration in anoles. Of the total 546 microRNA species from regenerating tail, adult brain and skeletal muscle, 350 are putatively novel and 196 are known microRNA precursors. Among these, three conserved microRNAs, including, miR-133a, miR-133b and miR-206, are crucial in tail 
regeneration by upregulating their target genes. They have also been reported to regulate regeneration and stem cell proliferation in other model systems including Zebrafish, Mouse etc. This indicates that mutations or losses of microRNA from the vertebrate lineage may be responsible for the differential capability of regeneration in vertebrates [38].

High expression of myogenic regulators represents dedifferentiation and structure formation of muscle. Furthermore, genes regulating dedifferentiation, muscle regeneration and axial development show changes in expression during tail regeneration in Anoles. Cells are found in regenerating tail muscle exhibit the properties of murine satellite cells. Therefore, source of tail muscle regeneration might be satellite cells [39]. Tail regeneration in Anolis lizards is tissue-specific through stem or progenitor cells; not like dedifferentiation and blastema-based model described in case of Salamander and Zebrafish [20].

\section{Comparison between Original and Regenerated Tail}

There is substantial difference between the original and regenerated body parts. New structure often differs from original structure. The appearance of original and regenerated tail is almost similar except skin color (due to lack of melanophore) and scale pattern (irregular) but internal structures are quite different. In green anoles, regenerated tail is the simplified version of original tail in terms of mechanism, function and structure $[2,40]$.

\subsection{Differences in Spinal Cord in Original and Regenerated tail}

In original tail, the autotomous region of caudal vertebrae is featured by a prominent neural spine and robust zygapophyses, which act as the attachment point for muscles and intramuscular septa, respectively. A well developed and complex spinal cord is found in original tail with segmental sensory, motor and autonomic nerves with organized dorsal root ganglia [31]. Peripheral nerves are detected nearby muscle bundles which is not originated from spinal cord through Gomorri's trichrome staining [41]. In regenerated tail, a defective spinal cord resides within an ependymal core (narrow lumen or central canal) providing axonal growth devoid of peripheral nerve or dorsal root ganglia [15, 31]. Uncovered axon clusters are present within the ependymal channel originated from the tail stump. This indicates that nerve fibers of regenerated tail are originated from ependymal rather than growing spinal cord's axons [16]. Ependymal core is further surrounded by a cartilage skeleton [15]. This regenerated cartilage tube contains vasculature within irregularly spaced foramina (passageways for blood vessels, increasing towards more distal regions [31]) but no nerves. A collagen rich perichondrium encasing both foramina and cartilage tube, indicates that foramina developed as a part of regenerative process [31].

\subsection{Muscular Difference in Original and Regenerated Tail}

Fully matured regenerated tails of green anoles comprise of spinal cord with hyaline cartilage tube, muscle with vasculature, and skin with scales [20]. In regenerated tails, muscles are irregular and elongated whereas muscles of original tails are arranged into nested cones forming myomeres attached to myosepta [14]. Regeneration of muscle tissues during tail regrowth in lizards is characterized by segmental myomeres [3] located beneath the dermis of new tail [42]. Unusual attachments among the irregular muscle bundles and cartilage tube is found in regenerated tail; while original tail retain muscles arranged in quadrants (package of four muscle bundles). Further, muscles within quadrants are attached to the vertebral column (via robust tendons) with regular patterns [21, 31] and cover central myoseptum (absent in regenerated tail) [31]. Abundance of connective tissue is also identified in regenerated tails than in original tail $[14,21]$.

\subsection{Functional Differences in Original and Regenerated Tail}

The structural difference between original and regenerated tails may contribute to major functional differences. In regenerated tail, a single cartilage tube replaces the interlocking segmental vertebrae of original tail. This could potentially reduce the capability of fine movement and flexibility 
as compared to original tail due to the lack of joint throughout the length of tail. In addition, potential range of motion might be affected due to lack of regularly spaced interdigitated muscle bundles found in original tail. Therefore, contraction of these irregular muscle bundle may produce gross movement but not fine movements of regenerated tail [13]. Abundance of fast myosin than slow myosin (via immunogold labeling) in myofibrils of regenerated tissues indicates limited blending capability of tails during locomotion and trashing after amputation [42]. A comparative analysis of intrinsic muscles of the original and regenerated tail predicts less coordinating capability, defective locomotion [21, 31]. Studies of the physical properties and range of motion of the original and regenerated tail are required to test this hypothesis. Therefore, from the anatomical and functional viewpoints, regenerated tail is an "imperfect replica" of the original tail. Most concerning imperfection is the skeleton that form unsegemented cartilage tube instead of vertebrae found in original tail [43].

\section{Factors Affecting the Tail Autotomy and Regeneration}

Tail autotomy occurs through biological friction and adhesion on the fracture planes of tail vertebrae. This is facilitated by the interlocking arrangement of tail muscles; which is easy and quick rather than previously assumed slow proteolysis of connective tissues $[1,44]$. Lizards help releasing their caudal limb by contracting their caudal muscles around those points. Reasonably, fractures occur at the nearby segment, or no more than three anterior fracture planes from there, where the lizard was attacked or poisoned [1]. It stipulates the importance of tails and severity of costs due to loss of tail. However, tail autotomy in leopard gecko, Eublepharis macularius, is not influenced by fracture planes or method of amputation; rather it is an intrinsic property of tail [21].

Regulations of some key proteins may involve with regeneration of tails in Anolis carolinensis because their expression during normal state and regenerating state are not same. During the tail regeneration of Anoles, proteins i.e., transferrin, nucleotide-binding domain of sugar kinase and CH1 domain in immunoglobulin are upregulated whereas proteins i.e., $\mathrm{T}$ complex protein-1, phosphoglucomutase-1, creatine kinase are downregulated [7].

Immunoblotting of telomerase- 1 component shows more telomerase activity in regenerating tail than in normal tissues. [45]. Hence, telomerase may also contribute to regeneration process in lizards. Furthermore, fat layer in tail may facilitate autotomy [1] in autotomous lizards, because of the presence of perivertebral adipose tissue deep in muscles and adjacent to the vertebrae unlike nonautonomous lizards [31]. Additional factors can contribute in the release of tails during predator attack including type of predators; age, size and sex [46] of the lizards; ecology and evolution of their ancestral lineage [22]. Evolution of caudal muscle, however, has been found to reduce the ability of caudal autotomy in some other lizards [47].

\section{Impact on Performance due to Autotomy and Regenerated Tail}

Lizard tail plays some vital functions such as locomotor performance (i.e., sprint speed, climbing speed, endurance speed), foraging, lipid storage, social status, attraction to partner etc. However, there is a significant cost due to the loss of tails [48-50]. It makes lizard more vulnerable to future attack [51]. Lizards control their body position in midair during jumping and falling by swinging through air and interacting with takeoff surface. Studies of ground reaction forces (GRFs), moment of inertia and center of mass (CoM) in lizards before and after autotomy showed that changes in these parameters may cause negative impacts on the body dynamics during jumping and falling [48, 49, 52]. For example, locomotive changes (i.e., morphology, GRFs and kinematics) in Eublepharis macularius due to autotomy, resulted in 13\% of anterior shift of CoM. This change, however, can be recovered partially by successful regeneration of tail [53]. Autotomy and regeneration, thus, maintain a variable relationship among morphology, kinematics and forces. Analysis of physical and mathematical model suggests that lizard tails act as an air stabilizer during jumping and falling [53]

Extrinsic muscles of tail are highly similar among different species of Anoles, including Anolis carolinensis, while arrangement of intrinsic muscles appears to be variable. Perhaps, variation in intrinsic muscle arrangement is responsible for the difference in locomotive behavior i.e., jumping 
and dashing ability instead of hind limb length. Since regenerated tail is an imperfect replica of original tail, anatomy of regenerated tail indicates that it would be less capable of fine movements as compared to original tail [13]. However, there is not enough experimental evidence to support it fully because tail loss in some terrestrial species is not associated with loss of running speed [13]. Prolactin increases the weight of regenerating tail through lipid deposition without affecting length but gonadotropin reduces the fat storage in fat bodies thereby causing weight loss [54]. Therefore, loss of fat bodies which could provide energy for new tail regrowth, may reduce the survival capacity in physiologically stressful conditions [51]. In fact, some species of lizard return to the location where the tail shed and eat the autotomized tail to indemnify the loss of fat reserve [50]. Since autotomy and regeneration of tail are studied in laboratory environment, not in real territory with others having original tail, there are possibilities that environmental and behavioral practices with lizards having original tail may compensate lost functionality [13].

\section{Future Perspective of Tail Regeneration}

In green anoles, regeneration is a highly ordered process utilizing initial developmental programs as well as regeneration to produce the correct types and pattern of cells required to sufficiently restore the structures and functions of the sacrificed tail [24]. The genes responsible for regeneration of tails in Anolis carolinensis promote chondrogenesis, myogenesis, and neurogenesis [20]. After self-amputation, lost tail is replenished by stem cells produced by dedifferentiation through regeneration process [2]. That is why potentials of stem cells in medical and health research are enormous [55].

Transcriptomic analysis showed that activation of Wnt signaling pathways are also needed during the regeneration of tails in green anoles [20]. Tadpoles are capable of tail regeneration but only at earlier stages not in later stages. Wnt and FGF signaling pathway are also necessary during muscle and spinal cord regeneration of wild tadpole, revealed by introducing transgenes for Dickkopf1 (dkk1). Dickkopf1 is an antagonist of Wnt signaling, i.e., the dominant negative form of the FGF receptor in wild tadpole. Precise regulation of bioelectrical signaling can induce tadpole tail regeneration in later stages. Like tadpole, humans are incapable of regeneration at their later stages. Genetic analysis of Anolis' regeneration thus may provide a clue about how and what types of regulations are needed to induce regeneration in other mammals or even in humans. [56, 57]. Three genes, responsible for an immune response, has been identified which impair the regeneration capability in Xenopus laevis in their refractory stage. Incorporation of FK506, an immunosuppressant, restores regenerative capability in refractory period, a stage deficient of regeneration capacity [58].

Therefore, future approaches for reactivating these signaling pathways, may provide a hope of getting back regenerative capability in human. It demands extensive interdisciplinary research approaches, such as advancement in gene therapy. However, questions arise how biophysical changes, i.e., transmembrane voltage gradients, ion concentration, control the regulation of transcriptional activation in the regenerative process [21]. To complete regeneration puzzle, we must find the answers to these questions.

\section{Conclusions}

Anolis carolinensis has been used as an excellent reptilian model for studying biological regeneration in all tetrapod vertebrates. Self-amputation and subsequent regeneration of lost tail is very common in green anoles. In fact, tail autotomy occurs as an anti-predation strategy to avoid or escape predator attack. This may provide a sudden benefit for survival or other fatal situation but also accompanied by numerous costs. Loss of tail affects the lizards in many aspects including locomotion, social status, mating attraction, fat storage etc. The genome sequencing of Green Anoles has greatly facilitated the study of tail regeneration. Conserved through the course of evolution, among the 326 genes involved in anoles' tail regeneration, 302 are common in human but in the state of switched-off. Also, three novel microRNAs are thought to play principal role to pursue tail regeneration in Anoles. Distribution of genetic makeup is very similar between green anoles and all other tetrapod vertebrates. As a result, there may scope to transform this miraculous natural 
phenomenon into common medical technology. Therefore, genetic analysis of these conserved genes, their repressed states, reactivation and regulation could improve biomedical science, for example, limb regeneration, genetic therapies for repairing lost limbs, organs, and nerves could be developed.

Acknowledgments: We would like to thank Kamal Khan and Ashekur Rahman for fetching up some pricey articles. Our special thanks go to Terrence B. Ritzman and Rebecca E. Fisher for permitting us to use some figures from their papers, and to John Wiley \& Sons, Inc. for their permission and support.

Author Contributions: Z.N. and A.I. both contributed on research and wrote the draft manuscript. S.H.C. and S.A. provided some important suggestions. All authors read and approved the final manuscript.

Conflicts of Interest: The authors declare no conflict of interest.

\section{Abbreviations}

The following abbreviations are used in this manuscript:

$\begin{array}{ll}\text { miR } & \text { MicroRNA } \\ \text { DPA } & \text { Days Post Autotomy } \\ \text { PWD } & \text { Post Wound Day } \\ \text { 5-BrdU } & \text { 5-Bromodeoxyuridine } \\ \text { 3H-T } & \text { Tritiated Thymidine } \\ \text { FGF7 } & \text { Fibroblast Growth Factor-7 } \\ \text { GRFs } & \text { Ground Reaction Forces } \\ \text { CoM } & \text { Center of Mass }\end{array}$

\section{References}

1. Sheppard, L.; Bellairs, A.d'A. The mechanism of autotomy in Lacerta. Brit. J. Herpetol. 1972, 4, $276-286$.

2. Gilbert, E.A.; Payne, S.L.; Vickaryous, M.K. The anatomy and histology of caudal autotomy and regeneration in lizards. Physiol. Biochem. Zool. 2013, 86, 631-644, DOI: 10.1086/673889, PMID: 24241061.

3. Bryant, S.V.; Bellairs, A.d'A. Autotomy and regeneration in reptiles. Biology of the Reptilia. 1985, 15, 301410.

4. Arnold, E.N. Caudal autotomy as a defense. Biology of the Reptilia. 1988, 16, 235-273.

5. Maginnis, T.L. The costs of autotomy and regeneration in animals: a review and framework for future research. Behav. Ecol. 2006, 17, 857-872, DOI: 10.1093/beheco/arl010.

6. Alibardi, L. Morphological and cellular aspects of tail and limb regeneration in lizards: A model system with implications for tissue regeneration in mammals. Adv. Anat. Embryol. Cell Biol. 2010, 207, 1-109, DOI: 10.1007/978-3-642-03733-7, PMID: 20334040.

7. Hong, V.; Thornton, B. Differential Protein Expression During Tail Regeneration of Anolis carolinensis. Research in Biology. 2015, Paper 3.

8. Higham, T.E.; Russell, A.P. Flip, flop and fly: modulated motor control and highly variable movement patterns of autotomized gecko tails. Biol. Lett. 2010, 6, 70-73, DOI: 10.1098/rsbl.2009.0577, PMID: 19740891, PMCID: PMC2817253.

9. Lovern, M.B.; Holmes, M.M.; Wade, J. The green anole (Anolis carolinensis): a reptilian model for laboratory studies of reproductive morphology and behavior. ILAR J. 2004, 45, 54-64, DOI: 10.1093/ilar.45.54, PMID: 14756155.

10. Alfoldi, J.; Di Palma, F.; Grabherr, M.; Williams, C.; Kong, L.; et al. The genome of the green anole lizard and a comparative analysis with birds and mammals. Nature 2011, 477, 587-591, DOI: 10.1038/nature10390.

11. W. L. Eckalbar, W.L.; Hutchins, E.D.; Markov, G.J.; Allen, A.N.; Corneveaux, J.J.; et al. Genome reannotation of the lizard Anolis carolinensis based on 14 adult and embryonic deep transcriptomes. BMC Genomics. 2013, 14, 49, DOI: 10.1186/1471-2164-14-49.

12. Tang, J.; Pecka, J.L.; Fritzsch, B.; Beisel, K.W.; He, D.Z.Z. Lizard and Frog Prestin: Evolutionary Insight into Functional Changes. PLoS ONE 2013, 8, e54388, DOI: 10.1371/journal.pone.0054388, PMID: 23342145, PMCID: PMC3546999. 
13. Ritzman, T.B.; Stroik, L.K.; Julik, E.; Hutchins, E.D.; Lasku, E.; et al. The gross anatomy of the original and regenerated tail in the green anole (Anolis carolinensis). Anat. Rec. (Hoboken) 2012, 295, 1596-1608, DOI: 10.1002/ar.22524, PMID: 22753107.

14. Tollis, M.; Hutchins, E.D.; Kusumi, K. Reptile genomes open the frontier for comparative analysis of amniote development and regeneration. Int. J. Dev. Biol. 2014, 58, 863-871, DOI: 10.1387/ijdb.140316kk, PMID: 26154326.

15. Cox, P.G. In vitro myogenesis of promuscle cells from the regenerating tail of the lizard, Anolis carolinensis. J. Morphol. 1968, 126, 1-17, DOI: 10.1002/jmor.1051260102.

16. Simpson Jr, S.B. Morphology of the regenerated spinal cord in the lizard, Anolis carolinensis. J. Comp. Neurol. 1968, 134, 193-210, DOI: 10.1002/cne.901340207, PMID: 5712416.

17. Simpson Jr, S.B.; Duffy, M.T. The lizard spinal cord: a model system for the study of spinal cord injury and repair. Prog. Brain Res. 1994, 103, 229-241, DOI: 10.1016/S0079-6123(08)61139-5, PMID: 7886207.

18. Alibardi, L. Development of the axial cartilaginous skeleton in the regenerating tail of lizards. Bull. Assoc. Anat. 1995, 79, 3-9, PMID: 7640411.

19. Alibardi, L. Muscle differentiation and morphogenesis in the regenerating tail of lizards. J. Anat. 1995, 186, 143-151, PMCID: PMC1167280.

20. Hutchins, E.D.; Markov, G.J.; Eckalbar, W.L.; George, R.M.; King, J.M. et al. Transcriptomic analysis of tail regeneration in the lizard Anolis carolinensis reveals activation of conserved vertebrate developmental and repair mechanisms. PLoS ONE 2014, 9, e105004, DOI: 10.1371/journal.pone.0105004, PMCID: PMC4139331.

21. Kusumi, K.; Fisher, R.E. Studying mechanisms of regeneration in amphibian and reptilian vertebrate models. Anat. Rec. (Hoboken) 2012, 295, 1529-1531, DOI: 10.1002/ar.22541, PMID: 22933304, PMCID: PMC3906853.

22. Higham, T.A.; Russell, A.P.; Zani, P.A. Integrative Biology of Tail Autotomy in Lizards. Physiol. Biochem. Zool. 2013, 86, 603-610, DOI: 10.1086/673875, PMID: 24241059.

23. Arnold, E.N. Evolutionary aspects of tail shedding in lizards and their relatives. J. Nat. Hist. 1984, 18, 127169, DOI: 10.1080/00222938400770131.

24. Capaldi, E.A.; Clause, A.R. Caudal autotomy and regeneration in lizards. J. Exp. Zool. A Comp. Exp. Biol. 2006, 305, 965-973, DOI: 10.1002/jez.a.346, PMID: 17068798.

25. Cohn, P.J. Tail Loss in Lizards. BIOSCIENCE 2009, 59, 728, DOI: 10.1525/bio.2009.59.8.23.

26. Cox, P.G. Some aspects of tail regeneration in the lizard, Anolis carolinensis I: A description based on histology and autoradiography. J. Exp. Zool. 1969, 171, 127-149, DOI: 10.1002/jez.1401710202.

27. King, R.S.; Newmark, P.A. The cell biology of regeneration. J. Cell Biol. 2012, 196, 553-562, DOI: 10.1083/jcb.201105099, PMID: 22391035, PMCID: PMC3307701.

28. Alibardi, L.; Celeghin, A.; Dalla, V.L. Wounding in lizards results in the release of $\beta$-defensins at the wound site and formation of an antimicrobial barrier. Dev. Comp. Immunol. 2011, 36, 557-565, DOI: 10.1016/j.dci.2011.09.012, PMID: 22001772.

29. Alibardi, L. Immunolocalization indicates that both original and regenerated lizard tail tissues contain populations of long retaining cells, putative stem/progenitor cells. Microsc. Res. Tech. 2015, 78, 1032-1045, DOI: 10.1002/jemt.22581, PMID: 26415629.

30. Egar, M.; Simpson, S.B.; Singer, M. The growth and differentiation of the regenerating spinal cord of the lizard, Anolis carolinensis. J. Morphol. 1970, 131, 131-151, DOI: 10.1002/jmor.1051310202, PMID: 5425076.

31. Fisher, R.E.; Geiger, L.A.; Stroik, L.K.; Hutchins, E.D.; George, R.M.; et al. A histological comparison of the original and regenerated tail in the green anole, Anolis carolinensis. Anat. Rec. (Hoboken) 2012, 295, 16091619, DOI: 10.1002/ar.22537, PMID: 22933242, PMCID: PMC4389898.

32. Alibardi, L. Original and regenerating lizard tail cartilage contain putative resident stem/progenitor cells. Micron. 2015, 78, 10-18, DOI: 10.1016/j.micron.2015.06.003, PMID: 26163156.

33. Alibardi, L.; Gibbons, J.; Simpson Jr, S. Fine structure of cells in the young regenerating spinal cord of the lizard Anolis carolinensis after H3-thymidine administration. Biol. Struct. Morphog. 1992, 4, 45-52, PMID: 1489867.

34. Wu, P.; Alibardi, L.; Chuong, C.M. Regeneration of reptilian scales after wounding: neogenesis, regional difference, and molecular modules. Regeneration (Oxf) 2014, 1, 15-26, DOI:10.1002/reg2.9, PMID: 24665362, PMCID: PMC3961719. 
35. Alibardi, L. Immunolocalization of FGF7 (KGF) in the regenerating tail of lizard suggests it is involved in the differentiation of the epidermis. Acta Histochem. 2015, 117, 718-724, DOI: 10.1016/j.acthis.2015.09.003, PMID: 26508592.

36. Alibardi, L. Immunolocalization of large corneous $\beta$-proteins in the green anole lizard (Anolis carolinensis) suggests that they form filaments that associate to the smaller $\beta$-proteins in the $\beta$ layer of the epidermis. J. Morphol. 2015, 276, 1244-1257, DOI: 10.1002/jmor.20415, PMID: 26220876.

37. Taylor, J.D.; Hadley, M.E. Chromatophores and color change in the lizard, Anolis carolinensis. Cell Tissue Res. 1970, 104, 282-294, DOI: 10.1007/BF00309737, PMID: 4193617.

38. Hutchins, E.D.; Eckalbar, W.L.; Wolter, J.M.; Mangone, M.; Kusumi, K. Differential expression of conserved and novel microRNAs during tail regeneration in the lizard Anolis carolinensis. BMC Genomics 2016, 17, 339, DOI: 10.1186/s12864-016-2640-3, PMID: 27150582, PMCID: PMC4858913.

39. Markov, G.J.; George, R.; Emmert, N.; Ammar, M.; Eckalbar, W.L.; Wade, J.; et al. Developmental gene activation in tail regeneration in the lizard, Anolis carolinensis. Dev. Biol. 2010, 344, 519-520, DOI: 10.1016/j.ydbio.2010.05.452.

40. Bryant, S.V.; Bellairs, A.d'A. Tail regeneration in the lizards Anguis fragilis and Lacerta dugesii. Zool. J. Linnean Soc. 1967, 46, 297-305, DOI: 10.1111/j.1096-3642.1967.tb00510.x.

41. Cox, P.G. Some aspects of tail regeneration in the lizard, Anolis carolinensis II: The role of the peripheral nerves. J. Exp. Zool. 1969, 171, 151-159, DOI: 10.1002/jez.1401710203.

42. Alibardi, L. Regenerating tail muscles in lizard contain Fast but not Slow Myosin indicating that most myofibers belong to the fast twitch type for rapid contraction. Tissue Cell. 2015, 47, 533-540, DOI: 10.1016/j.tice.2015.06.002, PMID: 26164738.

43. Lozito, T.P.; Tuan, R.S. Lizard tail regeneration: regulation of two distinct cartilage regions by Indian hedgehog. Dev. Biol. 2014, 399, 249-262, DOI: 10.1016/j.ydbio.2014.12.036, PMID: 25596336.

44. Sanggaard, K.W.; Danielsen, C.C.; Wogensen, L.; Vinding, M.S.; Rydtoft, L.M.; et al. Unique structural features facilitate lizard tail autotomy. PLOS ONE 2012, 7, e51803, DOI: 10.1371/journal.pone.0051803, PMID: 23284771, PMCID: PMC3526639.

45. Alibardi, L. Immunolocalization of the telomerase-1 component in cells of the regenerating tail, testis, and intestine of lizards. J. Morphol. 2015, 276, 748-758, DOI: 10.1002/jmor.20375, PMID: 25712511.

46. Irschick, D.J.; Vanhooydonck, B.; Herrel, A.; Meyers, J. Intraspecific correlations among morphology, performance and habitat use within a green anole lizard (Anolis carolinensis) population. Biol. J. Linnean Soc. 2004, 85, 211-221, DOI: 10.1111/j.1095-8312.2005.00486.x.

47. Zani, P.A. Patterns of caudal-autotomy evolution in lizards. J. Zool. 1996, 240, 201-220, DOI: 10.1111/j.14697998.1996.tb05280.x.

48. Gillis, G.B.; Kuo, C.Y.; Irschick, D. The Impact of Tail Loss on Stability during Jumping in Green Anoles (Anolis carolinensis). Physiol. Biochem. Zool. 2013, 86, 680-689, DOI: 10.1086/673756, PMID: 24241065.

49. Bonvini, L.A. Jumping behavior and the effects of caudal autotomy on performance in Anolis carolinensis. B. Sc. Honors, Mount Holyoke College, Massachusetts, USA, 2007.

50. Clark, D.R. Strategy of tail-autotomy in Ground Skink, Lygosoma laterale. J. Exp. Zool. 1971, 176, 295-302, DOI: 10.1002/jez.1401760305, PMID: 5548871.

51. Bateman, P.W.; Fleming, P.A. To cut a long tail short: a review of lizard caudal autotomy studies carried out over the last 20 years. J. Zool. 2009, 277, 1-14, DOI: 10.1111/j.1469-7998.2008.00484.x.

52. Gillis, G.B.; Bonvini, L.A.; Irschick, D.J. Losing stability: tail loss and jumping in the arboreal lizard Anolis carolinensis. J. Exp. Biol. 2009, 212, 604-609, DOI: 10.1242/jeb.024349, PMID: 19218510.

53. Jagnandan, K.; Russell, A.P.; Higham, T.E. Tail autotomy and subsequent regeneration alter the mechanics of locomotion in lizards. J. Exp. Biol. 2014, 217, 3891-3897, DOI: 10.1242/jeb.110916.

54. Licht, P. Interaction of prolactin and gonadotropins on appetite, growth, and tail regeneration in the lizard, Anolis carolinensis. Gen. Comp. Endocrinol. 1967, 9, 49-63, DOI: 10.1016/0016-6480(67)90096-2.

55. Cai, S.; Fu, X.; Sheng, Z. Dedifferentiation: A New Approach in Stem Cell Research. BIOSCIENCE 2007, 57, 655-662, DOI: 10.1641/B570805.

56. Lin, G.; Slack, J.M.W. Requirement for Wnt and FGF signaling in Xenopus tadpole tail regeneration. Dev. Biol. 2008, 316, 323-335, DOI: 10.1016/j.ydbio.2008.01.032, PMID: 18329638.

57. Levin, M. Bioelectric mechanisms in regeneration: Unique aspects and future perspectives. Semin. Cell Dev. Biol. 2009, 20, 543-556, DOI: 10.1016/j.semcdb.2009.04.013, PMID: 19406249, PMCID: PMC2706303. 
58. Naora, Y.; Kaneko, K.; Hishida, Y.; Fukazawa, T.; Kunieda, T.; Kubo, T. Analysis of the mechanisms that determine tail regenerative ability in Xenopus laevis tadpoles. Dev. Biol. 2010, 344, 519, DOI: 10.1016/j.ydbio.2010.05.375.

(C) 2016 by the authors; licensee Preprints, Basel, Switzerland. This article is an open access article distributed under the terms and conditions of the Creative Commons by Attribution (CC-BY) license (http://creativecommons.org/licenses/by/4.0/). 